\title{
ENFANTS ET ADOLESCENTS DE PARENTS SOUFFRANT DE TROUBLES PSYCHIQUES
}

Kurt Albermann, Brigitte Müller

Les enfants et adolescents de parents souffrant de troubles psychiques sont exposés à plus de pression et sont eux-mêmes plus à risque de développer des maladies psychiques. Mondialement sont concernés environ $15-23 \%$ des enfants, reporté à la Suisse cela représente environ 300'000 enfants et adolescents de moins de 18 ans $^{1,2)}$. Dans cet article nous décrivons les répercussions d'une maladie psychique d'un parent sur la santé des enfants et adolescents. Nous montrons aussi comment reconnaître ces situations et comment soutenir les ressources des enfants et des familles.

\section{Santé psychique}

La santé psychique n'est pas un état mais le résultat d'interactions multiples et dynamiques entre ressources et pressions auxquelles est soumis un individu. L'intensité et la fréquence des facteurs de stress et la vulnérabilité individuelle jouent un rôle autant que les ressources disponibles (forces, facteurs protecteurs) permettant de surmonter des situations de stress $^{3)}$. Dans le contexte de santé psychique de l'adulte, celle-ci est entre temps aussi définie en tant que bien-être («well-being»), avec ses différentes dimensions émotionnelles, psychiques et sociales ${ }^{4-6}$. Dans le meilleur des cas ces émotions positives comprennent le bonheur, la joie de vivre, le fait de reconnaître et exploiter ses capacités et des relations stables et bénéfique avec d'autres personnes, ainsi qu'une contribution à la société. Les enfants et adolescents dépendent, en fonction de leur stade de développement, de personnes qui les encadrent sensibles, aimantes et stables, de sécurité et d'un entourage stimulant. Cela leur permet de faire des expériences de vie qui correspondent à leur âge, d'où l'importance du contexte psychosocial à cet âge en quelque sorte «dépendant». Dans un modèle bio-psycho-social élargi ${ }^{7}$, la compréhension intégrative de la santé psychique comprend aussi des facteurs génétiques, épigénétiques et individuels ${ }^{7,8)}$.

\section{Troubles psychiques}

Les troubles psychiques compromettent la perception, la pensée et les émotions. Ils influencent le vécu et le comportement de la personne atteinte ${ }^{9}$, qui en souffre souvent elle-même. Cependant les enfants et adolescents, dépendant de leur âge, n'ont pas toujours (déjà) la capacité d'exprimer leurs perceptions et leurs sentiments de manière à ce que les parents ou leur entourage puissent les «lire et interpréter» correctement. Par ailleurs cela n'est pas toujours facile même pour des professionnels. Une vraie souffrance et la nécessité d'un traitement apparaissent en général lorsque l'enfant, les parents ou l'école les constatent, un changement du comportement habituel se manifeste ou si l'implication au quotidien ou les performances scolaires s'en ressentent. Le temps jusqu'à la consultation d'un professionnel est souvent très lourd à porter pour toutes les personnes concernées, le recours à une consultation, à des conseils et à un traitement est pour différentes raisons néanmoins bien trop souvent loin d'être optimal.

\section{Casuistique}

Il est donc facilement imaginable p.ex. que $\mathrm{M}^{\mathrm{me}}$. $\mathrm{R}$. avec une schizophrénie active ne soit que difficilement en mesure d'appréhender les besoins de ses deux enfants. Annina qui a dix ans et son frère Ben, de trois ans son cadet, ne savent pas comment comprendre les changements qui interviennent peu à peu dans le comportement de leur maman, ils sont déstabilisés et troublés, se sentent coupables. Ils ont honte lorsqu'ils font des courses avec leur maman et qu'elle parle de manière confuse, qu'elle craint les caméras suspendues au plafond et traverse le magasin avec le parapluie ouvert. Une nuit la police, alertée par un voisin, avait conduit la maman qui criait et chahutait menottée à la clinique psychiatrique. Les enfants ont peur, commencent à craindre leur maman, ils perdent pied. Le père est désespéré, ne sait plus comment faire, commence à boire et n'est guère un soutien pour ses enfants, d'autant plus qu'il a une position de cadre et dispose de peu de ressources en temps et personnelles. Il se gêne de demander à son employeur de la compréhension et de le décharger. Par la suite Ben qui est en première année primaire, devient énurétique et se met à se bagarrer avec les autres enfants. Le père décide de se séparer de sa femme, Annina s'occupe de Ben et du ménage, néglige ses hobbies et se distancie de plus en plus de ses amies. Plusieurs mois plus tard le père est invité à un entretien avec la direction de l'école: la moyenne d'Annina a chuté de plus d'un point, elle manque de concentration et est souvent distraite. (Figure 1)

\section{Prévalence}

D'après plusieurs études, la probabilité pour un enfant de développer un trouble psychique augmente d'un facteur de 3 à 7 s'il grandit dans une famille où l'un des parents a une maladie psychique. Les enfants

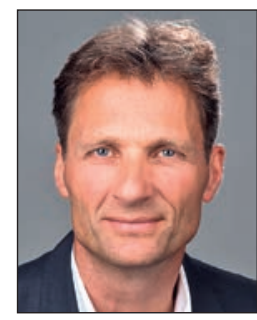

Kurt Albermann

https://doi.org/

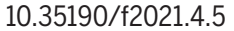

Correspondance: kurt.albermann@ksw.ch 


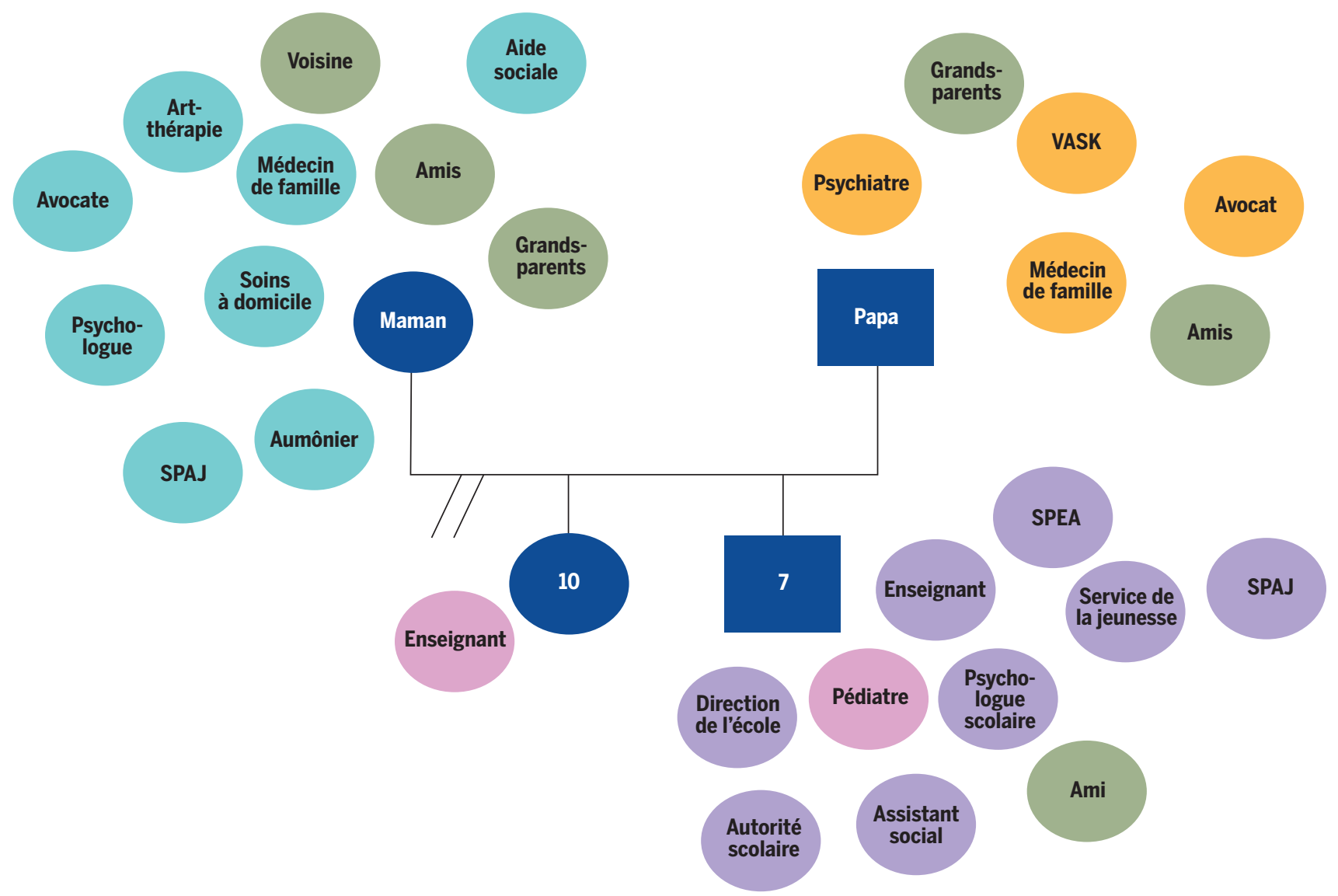

Figure 1. Représentation du système familial de la famille $R$. avec les deux enfants Annina (10 ans) et Ben (7 ans). La mère souffre de schizophrénie, le père s'en est séparé. La famille nécessite un important réseau de prise en charge, la mère est hospitalisée.

VASK: Association de proches des personnes souffrant de maladies psychiques

SPEA: Service de psychiatrie pour enfants et adolescents

SPAJ: Service de protection de l'adulte et de la jeunesse

qui sont pris en charge à cause d'un stress ou d'une maladie psychiques, sont souvent issus d'une famille où les parents aussi sont soumis à des stress ou sont atteints d'un trouble psychiques. Comparé aux enfants sans troubles psychiques, les enfants malades psychiquement sont nettement plus souvent issus de familles avec une dynamique familiale compliquée $(11.7 \text { vs } 28.3 \%)^{10}$. Le risque de maladie qu'encourt un enfant avec un parent schizophrène augmente de la moyenne dans la population générale de $1 \%$ à env.13\%. Lorsqu'un des parents souffre d'une schizophrénie, le risque à vie pour leurs enfants de contracter la même maladie atteint même env. $40 \%{ }^{11}$. Le risque de dépression lorsqu'un des parents souffre d'une maladie dépressive augmente de 2 à 6 fois. Environ $60 \%$ des enfants de parents avec une dépression développent pendant l'enfance ou l'adolescence un trouble psychique $^{12)}$. Une enquête représentative de 2017 en Suisse a montré que $7.9 \%$ des 3991 enfants et adolescents entre 10 et 15 ans questionnés, prennent en charge et assument les soins, régulièrement et de manière conséquente, d'un proche ${ }^{13)}$. Au niveau international on appelle les mineurs qui se trouvent dans une situation comparable young carers. Selon l'âge et la constellation familiale lors d'une maladie psychique des parents, ces jeunes aidants se recoupent avec les groupes mentionnés plus haut. En Suisse ils assurent souvent un soutien émotionnel, la prise en charge et les soins ainsi qu'une aide au ménage.

\section{Répercussions de la pandémie Covid-19 sur la santé psychique}

Le stress exceptionnel dû à la pandémie Covid-19 a fait perdre à de nombreuses familles l'équilibre entre ressources et facteurs de stress: soucis financiers, chômage, double charge par la garde des enfants et le travail à domicile engendrent plus de conflits, plus de pression sur la famille et ont, pour certaines, même menacé l'existence. Les stratégies d'adaptation établies ont été entravées par la limitation des échanges sociaux, la fermeture temporaire de sociétés, de structures culturelles et sportives et aussi des écoles. Les données sur l'utilisation de la «Main tendue», de Pro Juventute et les retours de pédopsychiatres montrent que les demandes de conseil en rapport avec des pensées suicidaires ont augmenté dans le contexte de la pandémi ${ }^{14)}$. Une enquête effectuée au Royaume-Uni a révélé que les enfants et adolescents avec un trouble de déficit d'attention/hyperactivité (TDA-H), un trouble du spectre autistique ainsi qu'avec des besoins pédagogiques particuliers ou issus de familles à bas revenu, récupéraient moins bien des restrictions dues au confinement et continuaient à manifester des symptômes de stress psychique ${ }^{15}$. D'après une en- 


\section{Formation continue}

quête anonyme en ligne réalisée par les Universités de Berne et Zurich, les enfants en âge préscolaire montraient, selon les indications des parents, plus souvent un comportement entêté et agressif, les 1119 ans par contre plutôt des problèmes psychiques comme des peurs ou dépressions. Pour les enfants de 1-10 ans, les troubles psychiques des parents sont liés à toutes les formes de problèmes psychiques de l'enfant. Alors que les enfants plus jeunes dépendent des stratégies d'adaptation, des ressources et de la situation socioéconomique des parents, les adolescents s'en sortaient souvent mieux grâce à leurs contacts sociaux avec des pairs ${ }^{16)}$. D'autres enquêtes montrent des réactions hétérogènes de la part des adolescents à la situation pandémique: certains réagissent avec un stress accru et des troubles de la concentration, à d'autres le confinement et la fermeture des écoles ont apporté une réduction du stress et de la pression sociale par les pairs, une amélioration du bien-être et plus de temps libre ${ }^{14,17)}$.

\section{Répercussions sur la famille}

Les familles avec un parent psychiquement malade représentent un groupe très hétérogène. Pour la famille et tout particulièrement pour les enfants, il est très important quel parent est malade, depuis combien de temps et de quelle manière la maladie se répercute sur les relations, la communication et l'organisation quotidienne de la famille. Les mères seules assument nettement plus souvent, malgré une maladie psychique, la garde des enfants que les pères seuls. II n'est pas rare qu'en plus elles doivent subvenir aux besoins financiers de la famille ${ }^{18)}$. Le séjour à l'hôpital d'une mère avec de petits enfants aura des conséquences considérables, notamment si elle élève seule ses enfants. Comme développé plus haut, le réseau familial est alors soumis à une pression extrême. Les grands-parents, des amis ou voisins ne sont pas toujours disponibles. Des structures d'aide à domicile, bénévoles ou professionnelles, et les services d'aide aux enfants et adolescents n'offrent pas toujours une prise en charge ininterrompue. II est donc parfois inévitable de devoir placer les enfants dans une famille d'accueil ou dans une institution.

\section{Pression sur les enfants et adolescents}

Les répercussions d'une maladie psychique ou d'une addiction parentale sont non-spécifiques. Dans le cadre des examens préventifs il est possible d'évoquer vis-à-vis des enfants, des adolescents et des parents d'éventuelles situations pesantes ou de stress. II est précieux de s'enquérir concrètement, dans une relation confidentielle, comment cela se passe au quotidien, quels changements les enfants et les parents souhaiteraient réellement, qui assume quelles tâches à la maison, si et comment ça fonctionne. Après une naissance un contact avec la consultation pour nourrissons ou la sage-femme peut s'avérer utile. Elles ont un accès direct au lieu de vie des familles et peuvent renseigner sur des situations difficiles et des besoins de soutien. La fréquence de la dépression postpartale est d'env. $15 \%$ pour les mères et d'env. $10 \%$ pour les pères. Il est important d'impliquer également les pères et de les d'inviter à des entretiens en commun. Les enfants de parents avec des troubles de la personnalité et des addictions sont les plus à risque. Surtout les enfants de mères avec des troubles de la personnalité borderline souffrent des conséquences de la maladie maternelle. Les enfants de parents avec une maladie psychique ont plus souvent des troubles du comportement et des troubles dans les domaines social, émotionnel et cognitif. Les jeunes aidants peuvent être soutenus de différentes manières, p.ex. directement par des explications et informations, des aides et conseils pratiques au quotidien, ou indirectement par des prestations à l'intention de la personne nécessitant un soutien ou au système familial tout entier $^{8)}$. Les jeunes aidants en Suisse expriment par ailleurs le souhait de plus de reconnaissance par les professionnel ${ }^{19}$ ). L'estime et la reconnaissance du travail et du soutien effectués réduisent le stress auquel sont soumis ces enfants et adolescents ${ }^{20}$.

\section{Ressources du réseau familial}

De nombreuses questions et incertitudes concernant l'organisation de la famille apparaissent lorsqu'un parent souffre d'une maladie psychique. Certaines familles peuvent avoir recours à un réseau privé ou public et obtenir un soutien adapté à leur situation. Cela peut fortement dépendre du stade de la maladie. Lors d'une première manifestation les ressources intrafamiliales sont souvent encore présentes. Dès que le processus devient chronique, le système de soutien familial s'épuise au fur et à mesure. La famille de parents seuls n'est souvent pas intégrée dans un système familial élargi, d'amis ou de voisinage, système qui fera donc aussi défaut lors de maladie, de séparation, de déménagement ou de problèmes financiers. Lorsque l'évolution d'une maladie psychique est chronique, les problèmes au niveau social, économique et familial deviennent de plus en plus complexes et les réseaux du domaine public par conséquent de plus en plus importants. Lorsque l'intégration dans un réseau est insuffisante ou absente, ou lorsque le parent malade ou, comme dans le cas décrit ci-dessus, toute la famille R. se replie sur elle-même, par honte, par méfiance due à la maladie ou suite à des expériences négatives, résulte une spirale négative avec une isolation sociale de plus en plus marquée.

\section{Qui parle aux enfants et adolescents?}

Partenaire, proches et enfants se trouvent souvent désemparés vis-à-vis d'une telle situation. Les enfants et adolescents souhaitent et doivent néanmoins recevoir des informations adaptées à leur âge et connaissances, et être impliqués dans la prise en charge souvent complexe de la maladie psychique de leur parent. Les enfants plus jeunes souhaitent être informés par le parent atteint, les adolescents par contre préfèrent l'implication et l'information par les professionnels, au cabinet médical ou à la clinique. Ils sont néanmoins souvent très critiques et craignent de ne pas obtenir des informations objectives ${ }^{21)}$. Pourtant les parents avec une maladie psychique, les familles et les enfants ne reçoivent généralement des informations appropriées ni pendant l'hospitalisation ni lors de la rentrée à domicile. Les médecins et le personnel soignant du système sanitaire pour adultes se sentent tenus 


\section{Formation continue}

d'informer en première ligne leur patient et moins les enfants, qu'ils ne connaissent souvent pas et qu'ils n'impliquent pas dans le traitement (figure 2) 2 $^{22,23)}$. II est donc indispensable d'aborder avec les parents leurs craintes et les besoins de leurs enfants, d'avoir des entretiens communs au sujet de la maladie psychique du parent et de répondre aux questions des enfants (tableau 1).

\section{Diagnostic et matériel}

II peut s'avérer utile de se servir de matériel d'information adapté à l'âge, de livres pour enfants sur le sujet, de marionnettes, peluches, brochures ou aussi de matériel filmé. Des questionnaires peuvent orienter sur le poids ressenti par les enfants. Pour un diagnostic plus approfondi on adressera l'enfant au psychologue ou pédopsychiatre. Avec le Strengths and Difficulties Questionnaire (SDQ) p.ex. on peut évaluer les forces et troubles du comportement d'enfants et adolescents de 4 à 16 ans. Les versions pour parents, enseignants et d'auto-évaluation pour adolescents du SDQ montrent une forte corrélation avec des questionnaires établis plus exhaustifs ${ }^{24,25)}$. Cinq minutes suffisent pour répondre. Les examens préventifs offrent au pédiatre la possibilité d'évoquer vis-à-vis des parents, en général la mère, les pressions individuelles et familiales subies et les facteurs de risque et protecteurs, et de mentionner les soutiens professionnels. Malheureusement il n'existe pas de remède miracle - pour chaque famille doivent être cherchés les moyens de soutien adaptés.

\section{Offres de soutien}

Entre temps existent dans toute la Suisse des structures où les enfants, les adolescents et les familles de parents avec une maladie psychique ou une addiction peuvent trouver conseil, traitement et soutien. Dans certaines structures psychiatriques pour adultes (p.ex. Königsfelden) ont été créées des consultations pour proches et enfants, collaborant étroitement avec les services de pédopsychiatrie (p.ex. Coire, Lucerne). II existe aussi des groupes de parents (p.ex. à Berne, Zurich, Winterthur) ou des offres intégrées parents-enfants pour mères avec nourrissons et petits enfants (p.ex. Affoltern) et pour mères/pères avec des enfants en âge préscolaire jusqu'à max. 5 ans (p.ex. Münsterlingen ${ }^{26)}$. Le canton Vaud a créé avec ZigZag Plus une structure proposant conseils et soutien aux enfants de parents avec une maladie psychique ou une addiction, dont l'établissement est prévu aussi dans les cantons de Fribourg, Neuchâtel et Valais. La fondation As'trame offre dans toute la Suisse romande entre autres du soutien pour les enfants de parents avec une maladie psychique. Certaines villes/cantons proposent des parrainages (p.ex. Bâle, Winterthur) ou un service SOS de garde d'enfants pour les familles en difficulté (région Winterthur), ainsi que des de ateliers de dessin ou groupes de discussion ou des conseils/ traitements individuels pour enfants de parents avec une maladie psychique ou une addiction (p.ex. Zebra, Windlicht à Winterthur). L'Association Dépression Postpartale Suisse informe sur cette maladie et s'engage à trouver le plus vite possible une aide adéquate pour les personnes atteintes.

Les familles dont un des parents est atteint d'une maladie psychique se différencient aussi par leurs besoins («needs»). Les besoins subjectifs peuvent différer sensiblement de ceux constatés par les professionnels. Des offres de soutien et de conseil ou des

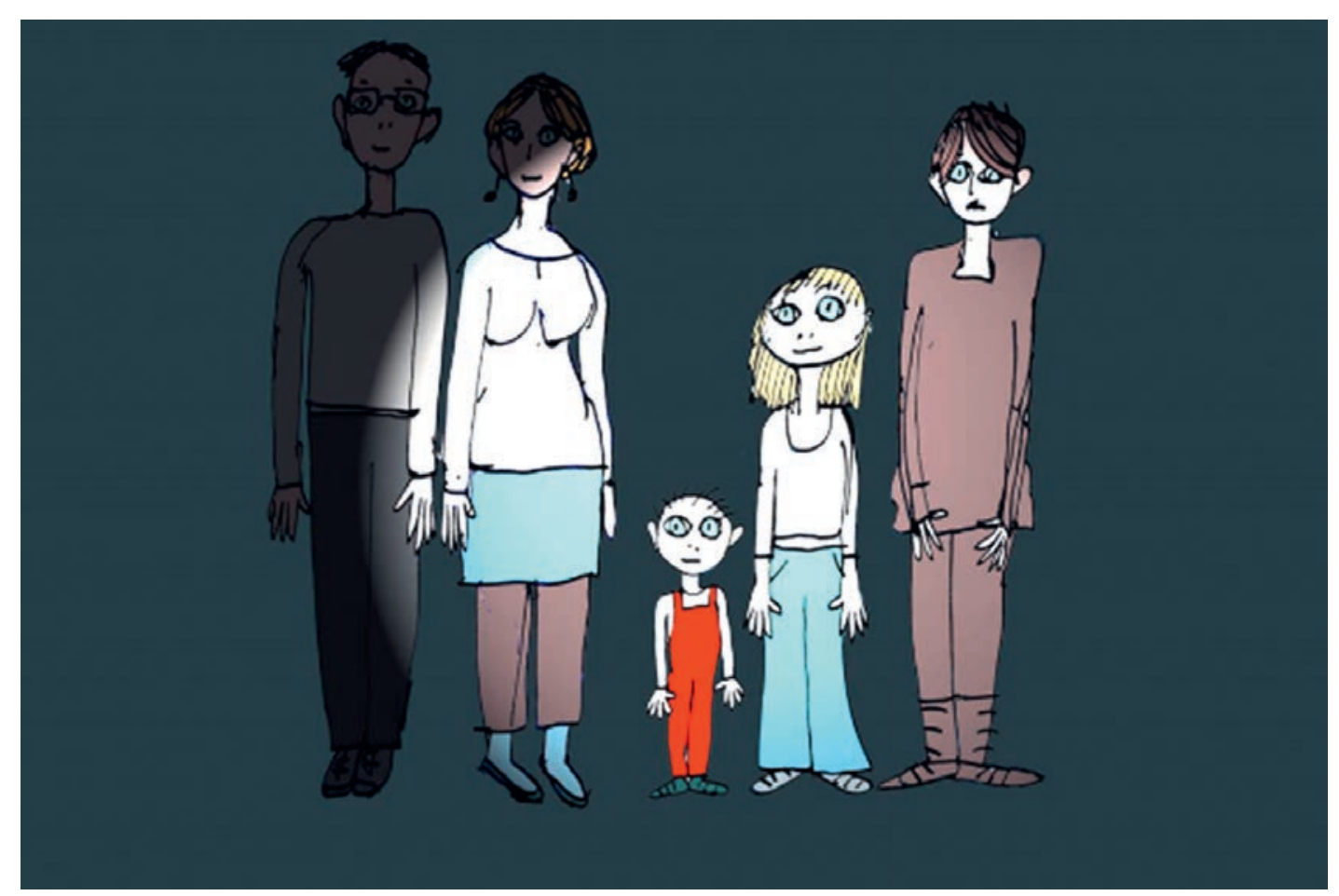

Figure 2. Le système de prise en charge des adultes est centré sur le patient individuel, donc sur le parent atteint (ici en noir). Parfois le parent sain est impliqué, rarement voire jamais les enfants. Pourtant ce sont les enfants qui ont besoin d'éclaircissements, d'implication et d'information (donc représentés ici en clair). www.liliancaprez.ch 


\section{Formation continue}

\begin{tabular}{l|l}
\hline $\begin{array}{l}\text { Comprendre ce qui s'est passé } \\
\text { (Causes de la maladie et évolution) }\end{array}$ & $\begin{array}{l}\text { Qu'est-ce qui se passe avec maman ou papa? } \\
\text { Pourquoi ils sont tombés malades? } \\
\text { Est-ce ma faute? Qu'est-ce que j'ai fait faux? } \\
\text { Ça s'arrangera? Combien de temps dure la maladie? }\end{array}$ \\
\hline $\begin{array}{l}\text { Comment dois-je me comporter? } \\
\text { (Vie familiale au quotidien) }\end{array}$ & $\begin{array}{l}\text { À quoi dois-je faire attention? Qu'est-ce que je dois faire } \\
\text { autrement? } \\
\text { Comment réagir au comportement qui a changé? } \\
\text { Que faire quand ça devient difficile à la maison? }\end{array}$ \\
\hline $\begin{array}{l}\text { Que vais-je devenir? } \\
\text { (Changements dans la vie familiale) }\end{array}$ & $\begin{array}{l}\text { Qui s'occupera de moi? } \\
\text { A qui puis-je faire confiance? Qui sera là pour moi? } \\
\text { Est-ce que je peux en parler? Avec qui? }\end{array}$ \\
\hline $\begin{array}{l}\text { Qu'est-ce que c'est d'avoir une maladie } \\
\text { psychique? } \\
\text { (Information sur les troubles psychiques) }\end{array}$ & $\begin{array}{l}\text { Que signifie en fait «psychiquement malade»? } \\
\text { Est-ce que ma maman / mon papa guérira? } \\
\text { Que puis-je faire? } \\
\text { Est-ce qu'il existe des médicaments? } \\
\text { Qu'est-ce qui se passe lors de la thérapie? } \\
\text { Puis-je aussi tomber malade? }\end{array}$ \\
\hline $\begin{array}{l}\text { Que faire si ...? } \\
\text { (Plan d'urgence 24/7) }\end{array}$ & $\begin{array}{l}\text { Qui m'aide? Qui puis-je appeler? } \\
\text { Où suis-je en sécurité? Où puis-je aller à quel moment? } \\
\text { Qui aidera alors ma maman / mon papa? } \\
\text { Dois-je appeler le 144 ou le 117? }\end{array}$ \\
\hline
\end{tabular}

Tableau 1. Besoins d'informations des enfants et adolescents (d'après Lenz et al. 2016²).

traitements à domicile peuvent soulager des familles concernées (p.ex. HotA/Argovie, MST-CAN/Bâle, Thurgovie). Le REPR (Relais Enfants Parents Romands, anciennement Carrefour Prison) accompagne les enfants de personnes incarcérées en Suisse romande, à la salle de visite ainsi qu'avant et après la visite, et soutient aussi les proches. Careum s'engage pour les droits et pour un soutien adéquat des jeunes aidants.

L'«Institut Kinderseele Schweiz» (iks) gère en tant que fondation suisse pour la promotion de la santé psychique des enfants et adolescents un site internet (en allemand) très complet avec des tuyaux, du matériel et des liens sur le sujet. Iks a produit de nombreux court métrages pour adolescents, parents et professionnels, qui informent sur différentes maladies psychiques et comment gérer des maladies psychiques et addictions parentales. En collaboration avec le canton de Thurgovie, iks a développé un outil de formation postgraduée en ligne pour collaborateurs de cliniques psychiatriques ou de services spécialisés, et avec le canton de St-Gall une plate-forme de contacts avec des adresses et des offres de soutien pour la Suisse alémanique. Enfin iks propose, avec des institutions partenaires, par chat en ligne, par téléphone ou sur place à Winterthur et Stäfa, des conseils et du soutien pour enfants, adolescents, parents et autres proches ainsi que pour les professionnels.

\section{Acceptation et autonomie familiale}

Pas toutes les familles nécessitent le même type de soutien - l'autonomie familiale doit être respectée dans tous les cas, et il faut discuter en commun, dans quels domaines est souhaité un soutien correspondant au mieux aux besoins de la famille. Certains parents sont bien informés sur les possibilités de soutien. Néanmoins ils craignent souvent que suite au contact avec le système de soutien on ne leur enlève les enfants pour les placer ${ }^{27)}$. II est souvent possible de gagner la confiance des parents en leur témoignant de la compréhension. II est utile de reconnaître qu'aussi des parents avec une maladie psychique ne veulent au fond que «le meilleur» pour leur enfant, qu'ils souhaitent qu'il puisse développer ses capacités et devenir une personne autonome et heureuse. Lorsque des parents ne peuvent plus assumer, en raison d'une maladie psychique ou d'une autre maladie sévère, toutes les tâches éducatives, il peut devenir nécessaire des transmettre cette responsabilité, temporairement ou à plus long terme, partiellement ou entièrement, à d'autres personnes. C'est pour cette raison que dans l'exemple de la famille $\mathrm{R}$. ont été instaurés une curatelle pour la mère et pour Ben ainsi que d'autres mesures de soutien. Ben et plus tard aussi Annina ont bénéficié d'une psychothérapie afin d'aborder les traumatismes vécus et leur permettre de gérer de manière adéquate la pression due à la maladie parentale et psychosociale. À l'école aussi ont eu lieu des entretiens afin de transmettre aux enseignants les informations nécessaires et leur permettre de soutenir au mieux les enfants (cf.à ce sujet $\left.{ }^{28}\right)$ ). Le père a réduit son travail, la mère vivait pour l'instant dans une communauté thérapeutique. Des mesures d'allégement et de soutien spécifiques permettent d'une part de réduire la souffrance, les maladies secondaires et à long terme souvent les coûts économiques, d'autre part d'améliorer sensiblement la qualité de vie des enfants et des parents. 


\section{Formation continue}

Les enfants nécessitent sécurité, des expériences relationnelles fiables et stimulantes ainsi qu'un entourage sûr et stable.

\section{Un soutien réussi}

Un soutien réussi s'oriente toujours au stade, à la gravité et aux symptômes de la maladie, ainsi qu'aux besoins d'information et aux stratégies d'adaptation de la famille et des enfants. Les familles concernées devraient avoir la possibilité de s'adresser, au sein du réseau, à leurs personnes de confiance (approche du «no wrong door»). Dans le cadre des examens préventifs, les pédiatres et médecins de famille ont la possibilité d'aborder avec les parents, généralement les mères, les difficultés familiales ou personnelles ainsi que les facteurs protecteurs et de risque. On peut évoquer des situations stressantes aussi directement avec les enfants et adolescents. Néanmoins ils ne donneront, par loyauté envers la famille et par crainte de conséquences imprévisibles, que rarement une réponse pouvant mettre en question la famille. De même on ne peut s'attendre à ce que les enfants ou des adolescents attirent l'attention à l'école (enfantine) sur le poids qu'ils portent.

Il est donc important de trouver conjointement aux parents et à l'école et en public, un langage approprié afin de répondre au tabou qui entoure les maladies psychiques. Ce n'est qu'ainsi que les services spécialisés et les personnes auxquelles les enfants font confiance pourront évoquer précocement les maladies psychiques en général et les maladies psychiques, physiques et les addictions des parents en particulier ${ }^{29)}$

\section{Mesures de protection de l'enfance}

Les enfants ne sont placés qu'en cas d'urgence extrême. Lorsque les familles n'arrivent plus à gérer seules les défis quotidiens, elles ont à disposition différentes offres à bas seuil pour des conseils éducatifs et du soutien ${ }^{8)}$. En outre dans la plupart des communes plus grandes existent des structures qui conseillent les enfants et adolescents et donnent des conseils éducatifs aux familles, ainsi que des services socioéducatifs scolaires ou des «aides éducatives» pour des enfants au besoins éducatifs et de prise en charge particuliers. Dans des situations familiales particulièrement problématiques est possible un accompagnement sociopédagogique au sein de la famille. II faudra toujours décider individuellement et en fonction de l'évolution, quelles mesures de soutien sont adaptées pour soutenir des parents psychiquement malades dans l'accomplissement de leur responsabilité éducative ${ }^{30}$ ). Les familles concernées souhaitent être activement impliquées et participer aux décisions prises à propos des éventuelles mesures de soutien, d'après la devise «Don't talk about us without us». Lorsqu'une mise en danger grave de l'enfant est imminente ou à craindre, des mesures immédiates pour sa protection sont indiquées ${ }^{31)}$. Si une décision concer- nant des mesures à long terme de protection de l'enfant, comme une curatelle, doit être prise par l'autorité de protection de l'enfance et de l'adulte, il est important pour la uréussite» de la mesure et l'atteinte des objectifs visés, que les parents et les enfants participent à la procédure. Les investigations nécessaires sont généralement faites per les services sociaux, les pédiatres et pédopsychiatres étant souvent consultés pour l'évaluation de questions spécifiques. Un projet du Fonds national suisse (FNS) examine actuellement comment les parents et les enfants peuvent être impliqués dans ces procédures et quels sont leurs désirs à ce propos ${ }^{32)}$

Les parents veulent en général «le meilleur» pour leur enfant. Cependant des troubles psychiques ou des addictions des parents peuvent temporairement ou durablement entamer leurs capacités éducatives et de discernement. Cela peut limiter sensiblement la capacité de garantir le «meilleur» ou le nécessaire au développement d'un enfant. Les attitudes et convictions familiales sont par ailleurs marquées par le contexte socioculturel et le conditions de socialisation de la famille.

\section{Collaboration coordonnée}

Les problèmes des familles avec un parent souffrant d'une maladie psychique ou d'une addiction sont souvent multiples, ce qui implique souvent différents professionnels et services spécialisés. La conséquence en est qu'un temps considérable est investi pour obtenir et échanger les informations entre partenaires du réseau. On visera donc une collaboration coordonnée entre partenaires au niveau du quartier, des institutions et de la commune ${ }^{33)}$. Cela implique une démarche transdisciplinaire et la reprise de perspectives et la collaboration entre disciplines, professions, organisations et structures de prise en charge partielle. La coordination et coopération peut se faire d'une part indépendamment du cas individuel par des structures du réseau, la planification des prises en charge et des organes responsables communs, d'autre part pour un cas spécifique par planification coordonnée des mesures de soutien et une gestion clarifiée du cas. Dans la mesure du possible, il faut viser une responsabilité commune et partagée ${ }^{34)}$. Pour une collaboration coordonnée, systématique et indépendante du cas individuel, la qualité et la fonctionnalité d'un réseau sont étroitement liées aux ressources personnelles, et donc aussi financières. Des dispositions de protection des données et de confidentialité peuvent limiter l'échange d'informations entre partenaires du réseau. Une levée pragmatique du secret médical/de fonction peut s'avérer utile, stipulant qui a été autorisé par les parents ou le détenteur de l'autorité parentale à contacter quelles personnes et à quel sujet l'échange d'informations est souhaité. Les expériences faites montrent qu'un engagement sans faille du réseau et des «porteurs d'idées» sont nécessaires pour créer et préserver à long terme un tel réseau.

\section{Conclusion}

La prise en charge réussie d'une famille avec des besoins complexes exige un soutien et une approche 


\section{Formation continue}

communs et professionnels ainsi que des échanges personnels à l'interne du réseau. Pour les enfants, les adolescents et les familles avec un parent souffrant d'une maladie psychique ou d'une addiction, la confiance, la continuité et la fiabilité sont des garanties importantes et déterminantes pour une collaboration réussie avec les professionnels. II est en outre nécessaire de sensibiliser davantage l'opinion publique, les professionnels, le monde politique et économique pour que la santé psychique puisse être évoquée plus naturellement et des éléments pertinents du bien-être puissent être intégrés au quotidien. Notamment dans les écoles il faut effectuer, conjointement aux enseignants, aux personnes d'encadrement, aux parents ainsi qu'aux élèves, un travail d'information concernant la santé psychique, surtout en termes de prévention et d'intervention précoce, en incluant au besoin le réseau professionnel scolaire et régional.

Pour la bibliographie, veuillez consulter notre version en ligne de l'article.

\section{Auteurs}

Dr. med. Kurt Albermann, Chefarzt, Sozialpädiatrisches Zentrum SPZ, Departement Kinder- und Jugendmedizin, Kantonsspital Winterthur Dr. med. Brigitte Müller, Psychologin FSP, Hochschule für Soziale Arbeit FHNW, Institut Kinder- und Jugendhilfe, Muttenz

Les auteurs n'ont déclaré aucun lien financier ou personnel en rapport avec cet article. 